\title{
Repair after nephron ablation reveals limitations of neonatal neonephrogenesis
}

\author{
Florian Tögel, ${ }^{1}$ M. Todd Valerius, ${ }^{1,2}$ Benjamin S. Freedman, ${ }^{1}$ Rossella latrino, ${ }^{1}$ Mor Grinstein, ${ }^{3}$ \\ and Joseph V. Bonventre ${ }^{1,2}$ \\ 'Renal Division, Brigham and Women's Hospital, Department of Medicine, Harvard Medical School, Boston, \\ Massachusetts, USA. ${ }^{2}$ Harvard Stem Cell Institute, Cambridge, Massachusetts, USA. ${ }^{3}$ Center for Regenerative Medicine \\ and Department of Orthopaedic Surgery, Massachusetts General Hospital, Boston, Massachusetts, USA.
}

The neonatal mouse kidney retains nephron progenitor cells in a nephrogenic zone for 3 days after birth. We evaluated whether de novo nephrogenesis can be induced postnatally beyond 3 days. Given the long-term implications of nephron number for kidney health, it would be useful to enhance nephrogenesis in the neonate. We induced nephron reduction by cryoinjury with or without contralateral nephrectomy during the neonatal period or after 1 week of age. There was no detectable compensatory de novo nephrogenesis, as determined by glomerular counting and lineage tracing. Contralateral nephrectomy resulted in additional adaptive healing, with little or no fibrosis, but did not also stimulate de novo nephrogenesis. In contrast, injury initiated at 1 week of age led to healing with fibrosis. Thus, despite the presence of progenitor cells and ongoing nephron maturation in the newborn mouse kidney, de novo nephrogenesis is not inducible by acute nephron reduction. This indicates that additional nephron progenitors cannot be recruited after birth despite partial renal ablation providing a reparative stimulus and suggests that nephron number in the mouse is predetermined at birth.

Authorship note: F. Toegel and M.T. Valerius contributed equally to this work.

Conflict of interest: The authors have declared that no conflict of interest exists.

Submitted: May 31, 2016 Accepted: December 13, 2016 Published: January 26, 2017

Reference information: JCI Insight. 2017;2(2):e88848. doi:10.1172/jici.nsight.88848.

\section{Introduction}

The number of nephrons in the human kidney, once established, does not increase during life but rather declines with age through attrition or disease. The "nephron endowment" is determined by 36 weeks of gestation (1), although new nephron formation after birth in humans has been suggested (2). Low birth weight (from any cause, including preterm birth) is strongly correlated with reduced nephron endowment $(3,4)$ and results in hyperfiltration from birth (5), leading to an increase risk of essential hypertension (6-9) and chronic kidney disease later in life $(10,11)$; these infants are thus placed at long-term risk for disease. Currently, there is no known method to increase nephron number in newborn or adult humans. Establishing an experimental system that induced neonephrogenesis postpartum would be an important first step in the development of mechanism-based therapeutics to augment nephron number in patients.

In the newborn mouse, nephron formation (nephrogenesis) has been postulated to continue until P3 (taking P1 as the day of birth), with the presence of Six $2^{+}$nephron progenitor cells and early nephron precursor structures, including renal vesicles, being seen as circumstantial evidence for de novo nephron formation (12). Fifty-one percent of mature nephrons are formed between P0 and P4, as shown in 3D modeling studies (13). Maturation of the nephrons continues after Six2 ${ }^{+}$cells are no longer present, and nephron growth accounts for the increase in kidney size. While de novo nephrogenesis is a possible contributor to kidney growth, an alternative explanation would be that the cap mesenchyme contains already fate-determined nephron progenitors that are set to complete nephrogenesis with the number of nephrons predetermined at birth by a multitude of different factors, including nutrition, oxygen tension, and genetics (14). This distinction is important because it has consequences for studies in regenerative medicine when evaluating the nephron-forming potential in the newborn mouse. Studies in the 1970s utilizing different nephron-counting procedures were discordant in demonstrating nephrogenic potential after birth with or without stimulation through contralateral nephrectomy (15). While the mouse model offers a postnatal window to investigate and potentially manipulate the final stages of nephrogenesis, formal lineage-tracing studies unequivocally demonstrating the extent of nephrogenesis after birth have never been conducted. The signals responsible for the cessation of nephrogenesis are unknown. It has been reported that BMP7 
pathway inhibition with LDN-193189, which blocks SMAD1/5, results in an extension of the time after birth that $\mathrm{CITED} 1^{+} / \mathrm{SIX} 2^{+}$nephron progenitors are present and an increase in nephron number (16). It is unclear whether this increase occurs with additional ureteric bud branching or a further increase in the number of nephrons per ureteric bud tip. Furthermore, it remains unclear whether the kidney has the ability to increase neonephrogenesis after birth when stimulated, for instance, following the reduction of kidney mass by cryoinjury. An evaluation of the endogenous ability of the kidney to modulate neonephrogenesis would offer potential paths for pharmacological interventions to prolong nephrogenesis in the premature birth neonate and potentially provide insight into how to replace nephrons in the adult.

In newborn animals, regenerative mechanisms have been reported for some organs. For example, newborn hearts in mice have been reported to regenerate cardiomyocytes and heal without fibrosis after resection of the tip, an ability that is lost in the first week after birth (17). Others, however, have challenged this observation (18). It has been proposed that organ formation and growth are regulated according to the size and functional demand of the newborn (19). Recent work suggests that nephron progenitors can be provided with signals that extend their self-renewal, raising the possibility of extending the neonephrogenesis in vivo (16). We hypothesized that reducing nephron mass during active nephrogenesis would alter this putative regulation, resulting in compensatory new nephron formation, a response that would be only possible during the early neonatal period when nephron progenitors were present.

To test this hypothesis, a model of cryoablation in newborn mice was developed to create a reproducible and localized injury to the kidney that reduces total kidney nephron mass. Acute reduction in nephron mass within 24 hours of birth resulted in an adaptive hypertrophic response and healing, with small amounts of fibrosis detected at 1 week (P7) and 3 weeks (P21). If contralateral nephrectomy was performed at the time of injury, the healing appeared more pronounced, with no signs of scarring by P21. By contrast, when cryoinjury-induced nephron reduction was performed at 1 week (P7), more extensive scarring was observed at P21. Genetic lineage-tracing analysis of the nephron progenitor cell population and marker analysis by immunofluorescence was done to assess the regenerative response of the newborn kidney. Whether the injury occurred within 24 hours of birth or at 7 days of age we detected no enhanced production of new nephrons, as measured by glomerular number, which remained reduced. Thus, the reduction of nephron number in the neonatal period when nephron progenitors are present does not stimulate de novo creation of additional glomeruli or other nephron segments. Rather, the newborn mouse responds to injury by compensatory hypertrophy of remaining nephrons.

\section{Results}

Cryoinjury to reduce nephron mass in newborn mice. In order to examine the regenerative potential of the newborn kidney, we developed a surgical approach using cryoinjury and nephrectomy. Newborn mice (we designate the day of birth as "P1," which includes the first 24 hours of life) were subjected to cryoinjury using a liquid nitrogen-cooled probe placed on the kidney surface for 3 seconds. This procedure led to a highly reproducible area of coagulative necrosis in the kidney (Figure 1, A and B). Twenty-four hours after injury, a necrotic area was documented involving approximately $15 \%-20 \%$ the size of the cortex and penetrating to the medullary area (Figure 1A). To assess the extent of injury beyond the necrotic area, we performed immunofluorescence analysis to detect KIM-1 (Havcr1), a marker of tubular injury (20), and LTL, a marker of proximal tubules (21). This revealed a penumbra of costaining $\mathrm{KIM}-1^{+} / \mathrm{LTL}^{+}$proximal tubular cells, indicating viable but injured tubules around the necrotic area (Figure 1, C and D).

Kidney architecture is restored after injury. In the mouse, it is generally accepted that the induction of new nephrons, or active nephrogenesis, continues to occur during the first 3 days after birth while the nephron progenitors are still present $(12,13,16,22)$. The timeline of our experiments, as indicated in Figure $1 \mathrm{E}$, was designed to evaluate whether there was potential for "de novo" induction of new nephrons in response to injury during this period. Mice were subjected to either newborn (P1) or late (P7) cryoinjury as a stimulus for repair processes that might include new nephron formation. Cryoinjury was performed on P1, when Six2 cells were present in the cap mesenchyme (Figure $1 \mathrm{~F}$ ). There was some variability in the exact timing of Six2 downregulation, likely due to the fact mothers delivered naturally during the night, resulting in a small range of delivery times. We examined the injury response through the first week (P3-P7) and at weaning age (P21). At P5, a time point 2 days after the Six $2^{+}$nephron progenitor cells have been exhausted during normal development $(12,13)$ and beyond the expected time of Six 2 positivity with normal variation, we observed a persistence of only a few dispersed Six $2^{+}$cells in a subset of cryoinjured mice (2 of 6) (Figure 1G). 

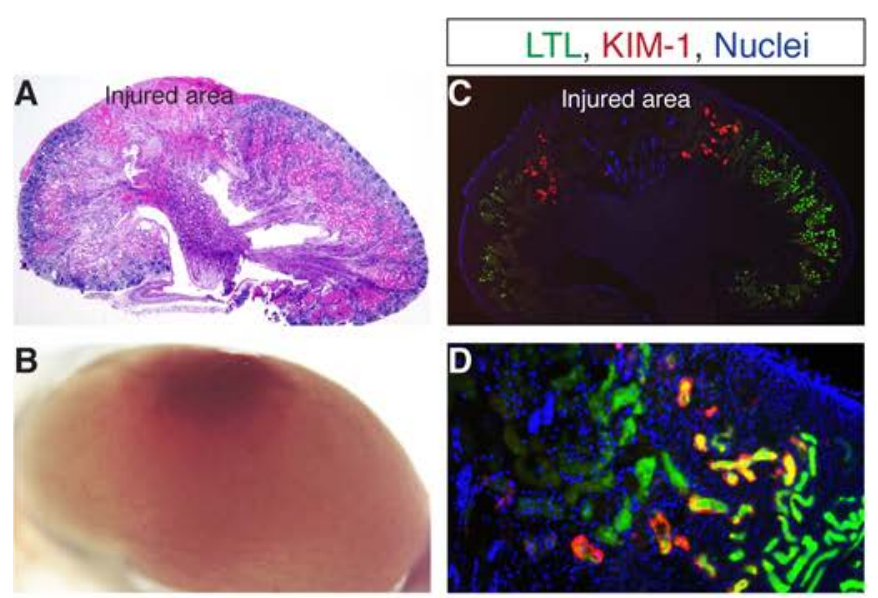

E

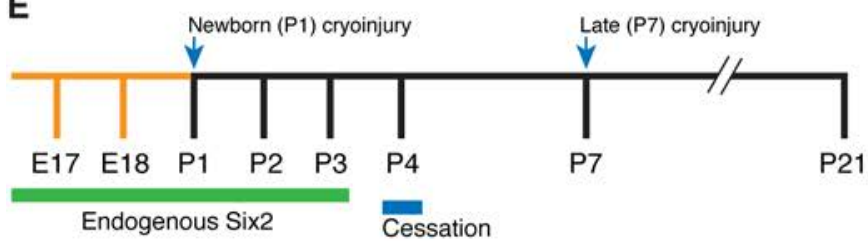

$\mathbf{F}$
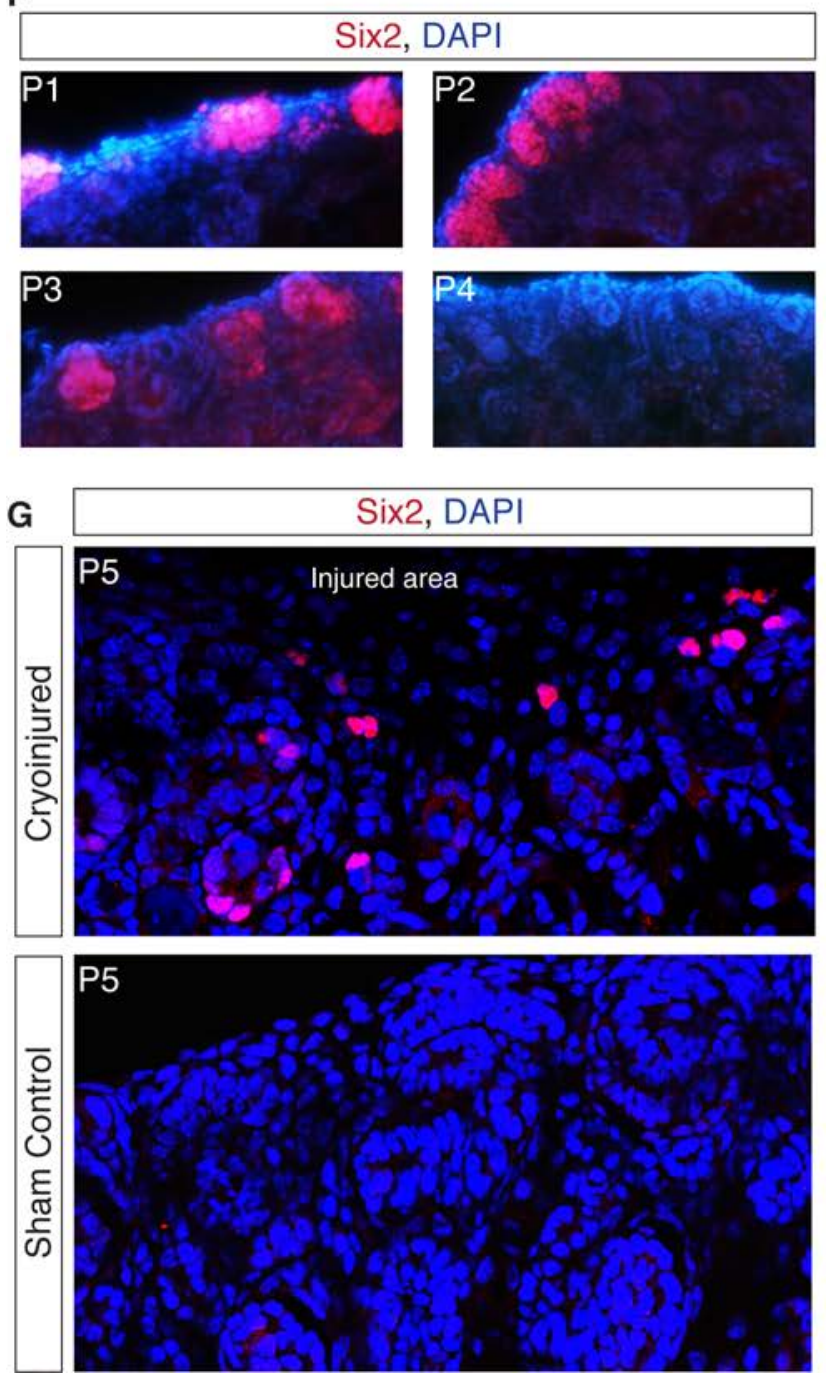

Figure 1. Kidney cryoinjury in newborns causes localized injury. (A) One day after injury, H\&E staining shows a necrotic area affecting 15\%-20\% of the kidney surrounded by intact renal parenchyma. Original magnification, $\times 3$. (B) Macroscopic view of the injury area 1 day after cryoinjury. (C) KIM-1 (Havcr1) expression (red fluorescence) 1 day after injury reflects tubular damage in the penumbral area around the necrotic injury. (D) A higher-magnification image (original magnification, $\times 20$ ) shows that KIM-1 is confined to tubular cells, as reflected by overlap of $\mathrm{KIM}-1^{+}$ (red) and $\mathrm{LTL}^{+}$(green) proximal tubules, resulting in yellow coloration. (E) Illustration delineating the time line of cryoinjury, Six2 expression, cessation of new nephron induction, and follow-up after injury. (F) Six2 is expressed until $\mathrm{P} 3$ in the nephron progenitors of the uninjured kidney cap mesenchyme. Original magnification, $\times 20$. (G) After cryoinjury, we observed dispersed Six2+ cells persisting beyond the normal $\mathrm{P} 3$ time of expression at the injury site in 2 of 6 animals examined for Six2 expression. Original magnification, $\times 100$.

Coagulative necrosis was apparent 1-day after injury. This was followed by reconstitution of the kidney architecture from the edges of the injury. This was discernible by day 3 after injury, with only a small residual indentation remaining on the kidney surface at the injury site at 1 week of age (P7) (Figure 2A). The weights of the injured left kidneys (normalized to body weight) were initially not significantly different from the weights of the contralateral control kidneys (Figure 2B) but started to lag behind the control kidneys 3 days after injury and remained decreased compared with contralateral controls on P8 and P21 (Figure 2, B and $\mathrm{C}$ ), despite gross anatomical restoration of tissue architecture. Uninjured contralateral control kidneys did not undergo significant hypertrophy, as reflected by kidney-weight-to-body-weight ratios that were similar to sham-operated kidneys. The lack of detectable hypertrophy in the contralateral kidney was also seen when animals were cryoinjured on P7 (Figure 2C).

We hypothesized that the continued presence of an uninjured contralateral kidney might inhibit the regenerative response. To investigate this, we performed unilateral nephrectomy with or without cryoinjury. Unilateral nephrectomy in newborn animals without cryoinjury caused compensatory hypertrophy in the kidney, as indicated by an increased kidney-weight-to-body-weight ratio (compare sham with sham/nephrectomy in Figure 2C). Unilateral nephrectomy at the time of cryoinjury also resulted in compensatory hypertrophy in the injured kidneys, as indicated by a kidney-weight-to-body-weight ratio that did not significantly differ from that of uninjured kidneys after contralateral nephrectomy (Figure 2, C and D). The total nephron number 1 week after newborn cryoinjury was determined by glomerular counting using the acid maceration method $(23,24)$. This method is based on glomerular counting of a sample of whole kidney tissue to minimize bias. An example of a sample used for counting is shown in Supplemental Figure 1 (supplemental material available online with this article; doi:10.1172/jci.insight.88848DS1). The injured kidneys had a significantly decreased number of total glomeruli compared with contralateral control kidneys (Figure 2E). Sham animals had glomerular numbers comparable to those of the uninjured contralateral control kidneys, without a significant difference between the right and left kidney. In addition, contralateral 
A

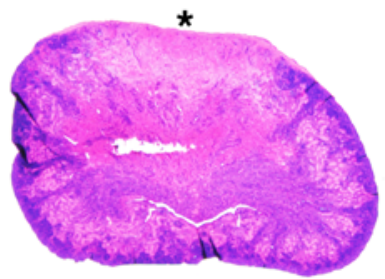

Newborn cryoinjury day 1 post injury

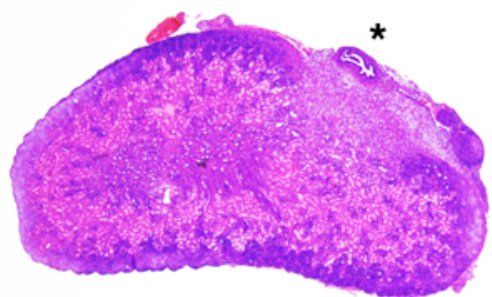

Newborn cryoinjury day 3 post injury

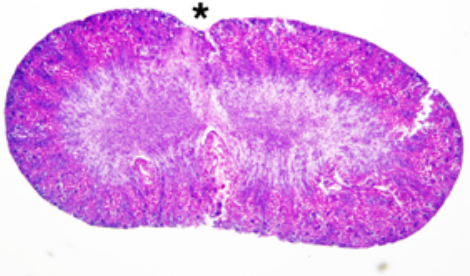

Newborn cryoinjury day 7 post injury
B

Kidney weight after injury

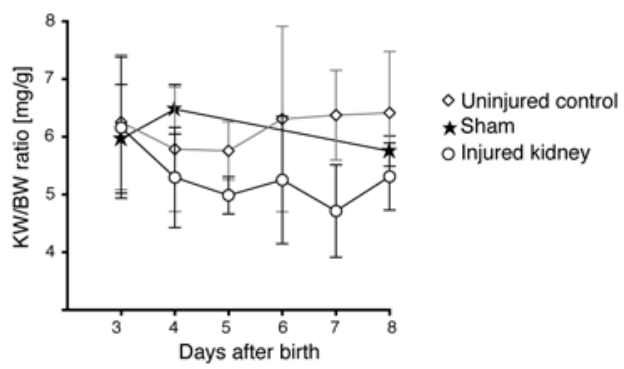

D

$$
\begin{gathered}
\text { Kidney weight one week } \\
\text { post P1 injury }
\end{gathered}
$$

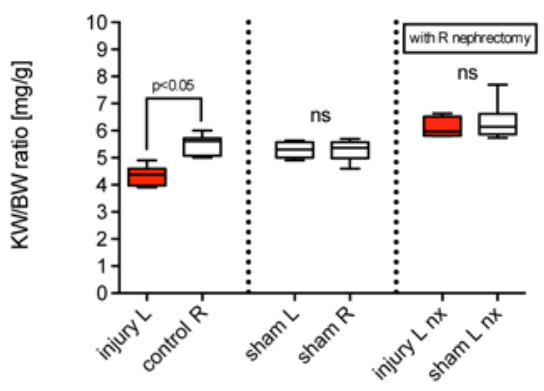

$\mathbf{F}$

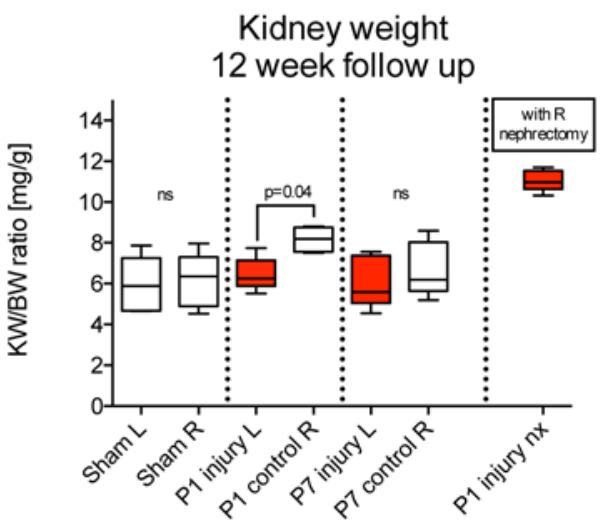

C

Kidney weight after injury at P21

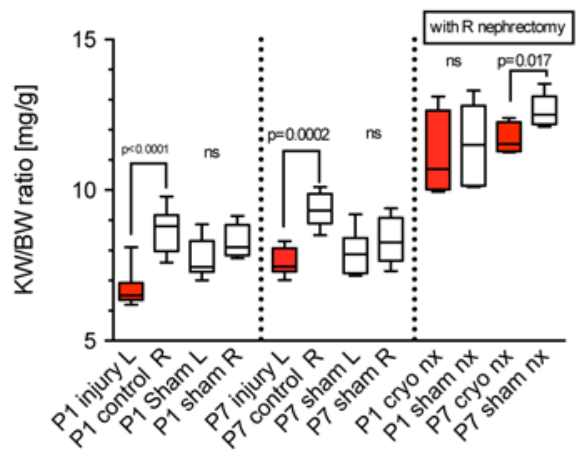

E

Glomerular count one week post $\mathrm{P} 1$ injury

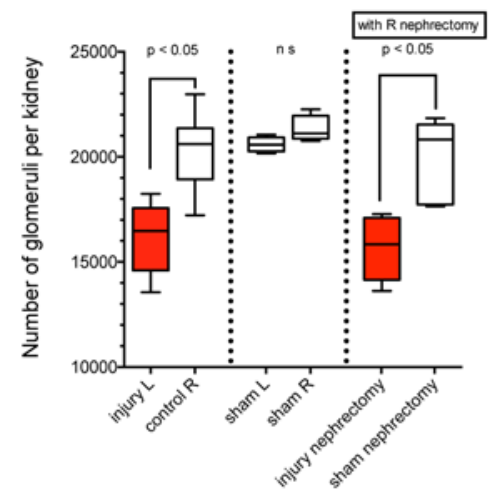

G

Neonatal resections

kidney weight 5 weeks post injury

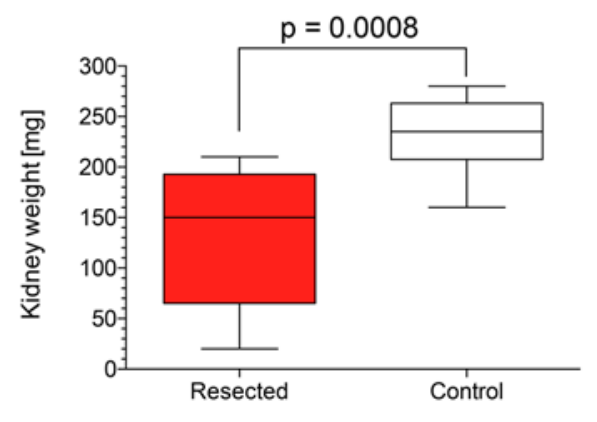

Figure 2. Cryoinjured kidneys exhibit reduced kidney mass and glomerular number. (A) Paraffin sections of kidney parenchyma after cryoinjury, with contralateral kidney intact, show a progressive size reduction in the injured necrotic area (asterisks) compared with the surrounding kidney parenchyma. On P7, there was resolution of the injury, with only a small indentation remaining at the site of the injury. Original magnification, $\times 3$. (B) Kidney weight progressively declined in the first 7 days after injury compared with that in uninjured control kidneys and sham controls. $n=104$ animals, $n>6$ per time 
point. (C) Kidney weight lagged significantly behind in injured kidneys on P21 in animals injured at birth (P1) and injured 1 week after birth (P7). Animals subjected to contralateral nephrectomy with injury had the same kidney weight as sham animals subjected to nephrectomy, indicating a hypertrophic response after contralateral nephrectomy. $n=58$ animals, $n>5$ per time point. Ordinary 1-way ANOVA, Dunnett's multiple comparison test. (D) Kidneyweight-to-body-weight ratio was significantly different at 1 week after injury without contralateral nephrectomy. When injury occurred with contralateral nephrectomy, there was not a significant difference in ratios after 1 week. $n=31$ kidneys. Ordinary 1-way ANOVA, Tukey's multiple comparison test. (E) Glomerular number, as determined by the acid maceration method, is significantly decreased 1 week after cryoinjury in injured kidneys compared with contralateral control kidneys and sham animals. $n=31$ kidneys. Ordinary 1-way ANOVA, Tukey's multiple comparison test. (F) Long-term (12 week) follow-up of kidneys subjected to cryoinjury shows the same weight ratio pattern in injured kidneys, which weighed significantly less compared with noninjured controls or shams. $n>5$ animals per group. Ordinary 1-way ANOVA, Tukey's multiple comparison test. (G) Kidney mass reduction at P1 by resection of one kidney pole. The mass of the injured kidney remains reduced compared with contralateral controls at 5 weeks after injury. All data include the mean \pm SD. In box-and-whisker plots, whiskers depict the minimum and maximum values, boxes depict 25 th to 75 th percentiles, and lines depict the median.

nephrectomy did not affect glomerular number in injured kidneys, as there was an equivalent decrease in glomerular number despite an increased kidney weight. Overall, the absence of an increase in glomerular counts indicated compensatory hypertrophy rather than new nephron formation as the mechanism for restoration to normal kidney weight (Figure 2D).

To determine the long-term implications of the cryoinjury and subsequent whole kidney development after injury, animals were followed into adulthood and euthanized at 12 weeks. The kidney-weightto-body-weight ratio increased with age in both injured and sham controls. The differences in kidneyweight-to-body-weight ratios observed at 1 week after injury were still evident at 12 weeks after left kidney injury (Figure 2F). Additionally, we examined kidneys subjected to resection of a kidney lower pole as a method to reduce nephron mass at birth. At 5 weeks after resection, kidney weight of the resected kidneys remained reduced compared with contralateral controls (Figure 2G), indicating a complete lack of a compensatory response to this type of injury also.

An increase in the kidney-weight-to-body-weight ratio after injury could be caused by a hypertrophic response solely, increased epithelial cell proliferation, or de novo nephron formation in response to injury. Having ruled out the last possibility, we examined the proliferative response by staining for Ki67 to determine if accelerated proliferation accounts for restitution of kidney architecture after cryoinjury (Figure $3 \mathrm{~A})$. The number of proliferating cells in the area of cortex or outer medulla surrounding the injury was not significantly different between injured kidneys and uninjured contralateral controls or sham controls on P3, P4, P6 and P8, which is consistent with a hypertrophic, rather than hyperplastic, response to the injury (Figure 3B). This hypertrophic response is greater if the contralateral kidney is removed.

Developmentally regulated genes are not altered in response to cryoinjury. Expression patterns of master regulator genes expressed during kidney development were assessed by immunofluorescence and in situ hybridization to identify cells engaged in nephrogenesis. The transcription factor Pax 2 is expressed during nephrogenesis and regulates ureteric bud branching and outgrowth (25). The pattern of expression of Pax2 in injured and contralateral control kidneys from P3 to P6 was not affected by cryoinjury. Pax2 expression was seen in cap mesenchyme cells in the subcortical area as well as in collecting duct cells extending into the medulla (Figure 4A). Lhx1 plays an essential role in nephrogenesis and is expressed in the intermediate mesoderm and in the nephric mesenchyme (26). After cryoinjury, $L h x 1$ transcripts are equally expressed in injured and contralateral control kidneys in the cap mesenchyme until P6, with no expression at P7 or later in injured kidneys, contralateral kidneys, and sham control kidneys (Figure 4B).

Persistent Six $2^{+}$cells at the injury site do not form additional new nephrons. To determine the fate of Six $2^{+}$nephron progenitors after cryoinjury, we used the tamoxifen-dependent Cre line, Six $2^{\mathrm{GCE}}$ (27), to lineage trace these cells. Six $2^{\mathrm{GCE} /+}$ heterozygous mice were crossed to the Cre-responsive R26R ${ }^{\text {tdTomato/dtomato }}$ homozygous reporter mice, resulting in Six2 ${ }^{\mathrm{GCE} /+} ; \mathrm{R}^{26 \mathrm{R}^{\mathrm{tdT} T o m a t o} /+}$ double heterozygous animals and R26R ${ }^{\mathrm{td} T o m a t o /+}$ littermate controls (Figure 5A). Tamoxifen dosing was used to label Six $2^{+}$cells prior to or during injury, permanently labeling Six $2^{+}$ descendant daughter cells from that time forward with tdTomato expression. Glomeruli that were tdTomato ${ }^{+}$ were therefore formed after tamoxifen-induced labeling of the Six $2^{+}$nephron progenitors. We measured the percentage of glomeruli formed after tamoxifen induction by staining all glomeruli by immunofluorescence (Figure $5 \mathrm{C}$ ) and determining the number of tdTomato/podocalyxin double-positive glomeruli. When control pregnant mice were administered tamoxifen at E17.5, approximately 50\% of glomeruli were labeled with tdTomato when examined on $\mathrm{P} 7$, indicating that the contribution of Six $2^{+}$progenitor cells at day 17.5 is due to ongoing nephrogenesis (Figure 5B). R26R ${ }^{\text {tdTomato/+ }}$ littermate controls given tamoxifen did not show any labeling. Thus, about half of nephrons are formed after E17.5 from the Six2+ cap mesenchyme in our system, approximating the 
A

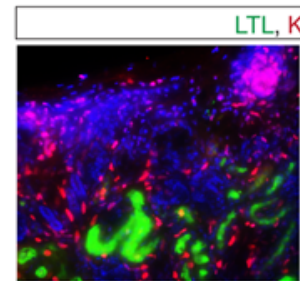

Injury area

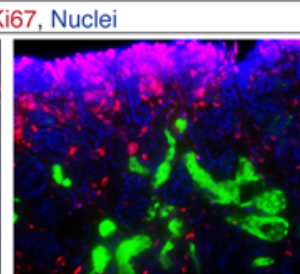

Remote from injury
B

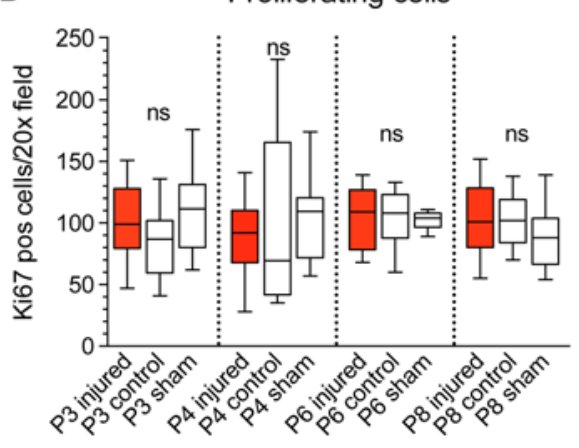

Figure 3. No increase in proliferation with cryoablation. (A) Numbers of proliferating cells (Ki67 positive, red) were not different around injured areas compared with remote cortical areas of the injured or contralateral control kidneys as well as sham animals of the same age. Original magnification, $\times 20$. LTL+ proximal tubules, green. (B) Analysis of the number of proliferating cells per high-power field (original magnification, $\times 20$ ) was done at P3, P4, P6, and P8 and showed no significant difference between injured kidneys, sham operated kidneys, or contralateral control kidneys (10 high-power fields, $n=3-5$ animals per group). In box-and-whisker plots, whiskers depict the minimum and maximum values, boxes depict 25th to 75th percentiles, and lines depict the median. Ordinary 1-way ANOVA, Tukey's multiple comparison test.

literature (28). When tamoxifen was administered on P1, approximately $45 \%$ of glomeruli, together with surrounding tubules, were labeled with tdTomato (Figure 5C). If the cryoablation injury caused persistent or prolonged nephron progenitor maintenance (now labeled) and nephron induction, then we would predict an increase in the percentage of tdTomato/ podocalyxin double-positive glomeruli. The number of labeled glomeruli in cryoinjured kidneys, however, remained statistically unchanged at approximately $46 \%$ when compared with contralateral controls when kidneys were subjected to cryoinjury on P1 with immediate injection of tamoxifen after injury (Figure 5F). Thus, consistent with our results, we did not detect an increase in the rate or duration of nephron formation after injury by labeled nephron progenitor fate tracing.

We next determined the fate of the rare dispersed Six $2^{+}$cells found in the area adjacent to the injury by labeling Six $2^{+}$cells 2 days after injury. If the dispersed Six $2^{+}$cells entered into a nephrogenic program, we would predict fully labeled nephrons to form. However, in animals subjected to cryoinjury on $\mathrm{P} 1$, with tamoxifen injected at the end of nephrogenesis (P3-P4, $n=4$, data not shown), there were similar amounts of Six2-derived tdTomato ${ }^{+}$single cells in the outer cortex of cryoinjured and contralateral kidneys. (Figure 5, D and E, right). No increase in the number of completely labeled tdT ${ }^{+}$ nephrons was present, indicating absence of de novo nephrogenesis. tdTomato ${ }^{+}$cells were scattered as single cells within glomeruli and tubules of nephrons, indicating that $\mathrm{Six}^{+}$cells were already lineage committed and not truly multipotent anymore, giving rise only to parts of nephrons without stem cell self-renewal.

Increased development of fibrotic lesions after injury in 1-week-old (P7) mice. To characterize the development of fibrosis following cryoinjury, we examined kidneys at 3 weeks and 12 weeks after injury induced at either P1 or P7, with or without contralateral nephrectomy. When cryoinjury was performed on P1 newborns without contralateral nephrectomy, the injured kidneys recovered either without noticeable surface abnormalites or had, in about $50 \%$ of the cases, a small residual macroscopic indentation at the site of cryoinjury 3 weeks after surgery (Figure 6A). Microscopically, Sirius red-stained wedges were observed at the injury site, with an indentation on the cortical surface. When contralateral nephrectomy was performed at the time of injury, fibrosis was not detected when injury was induced in P1 newborns (Figure 6A, 3 weeks, $\mathrm{P} 1$ cryoinjury/nx), indicating that contralateral nephrectomy prevented the development of fibrosis. In contrast, when injury was induced on P7, macroscopic injury persisted to week 3, even with contralateral nephrectomy (compare Figure 6A, P1 cryoinjury/nx with P7 cryoinjury/nx). In addition, Sirius red staining and $\alpha$-SMA staining at 3 weeks were increased in the P7 injured kidneys in the presence of contralateral nephrectomy, indicating that contralateral nephrectomy did not prevent fibrosis when injury occurred at the P7 time point. When P1 and P7 injured animals were examined at a longer time point (12 weeks after injury), a region of cortical thinning was noted in all kidneys and a persistent trichrome ${ }^{+}$area remained in the cortex at the injury site in approximately one-third of the P7 injured animals (Figure 6B).

\section{Discussion}

Nephrogenesis necessitates the interplay of different progenitor cell populations in a coordinated fashion, with reciprocal induction and maturation into various differentiated cell types. It is estimated that there are 26 highly specialized cell types populating a nephron, making nephrogenesis one of the most detailed and specialized processes in organogenesis (29). Factors that govern the final number of nephrons at birth are unknown, but in humans low nephron endowment correlates with low birth weight, female gender, short adult stature, and prematurity (30). While in vitro approaches for the differentiation of stem cells to kidney structures are showing promise (31-34), facilitating nephrogenesis in vivo would be a highly desirable and efficient tool in treating disease. The de novo generation of nephrons to substitute for the low nephron 


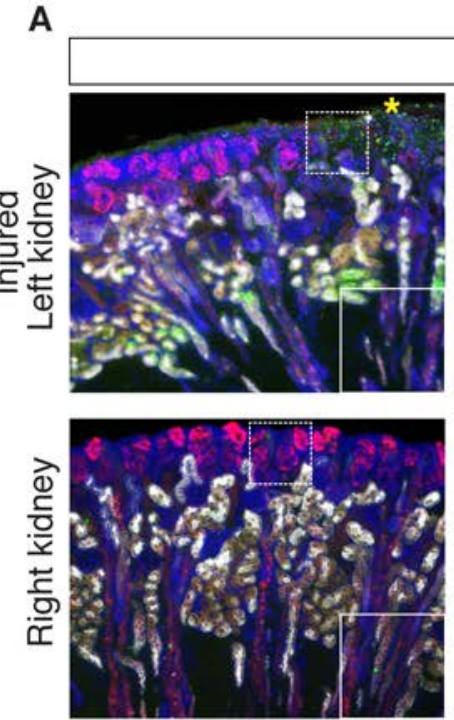

P3
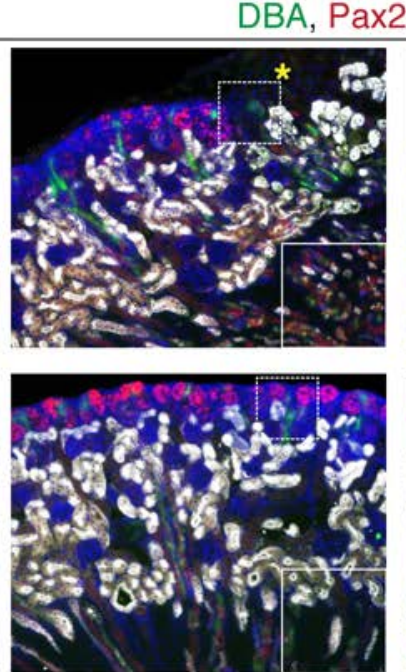

P4
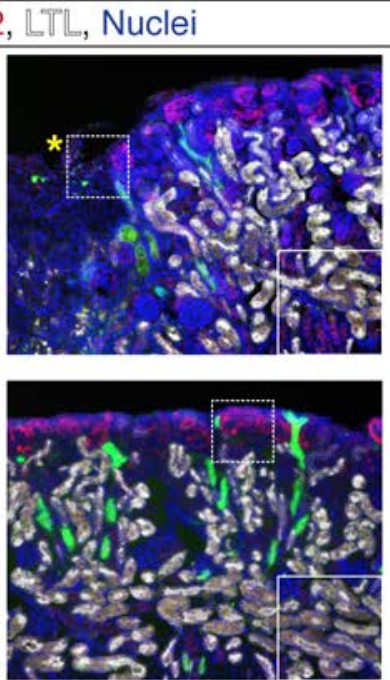

P5
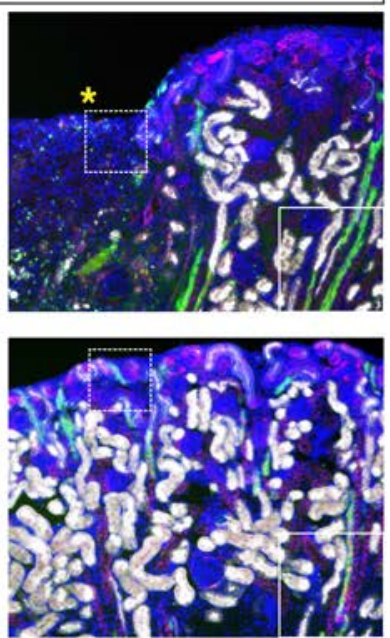

P6

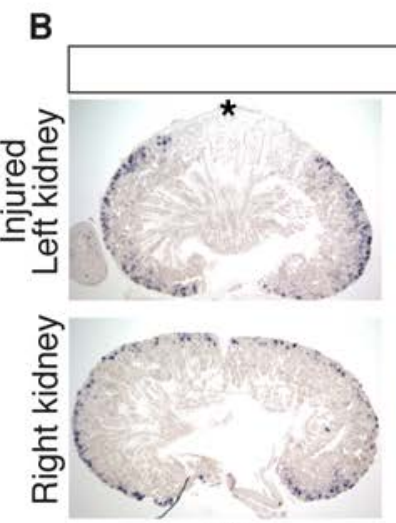

P3

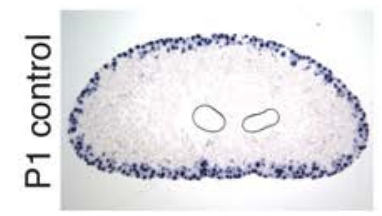

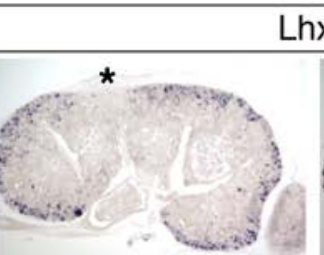

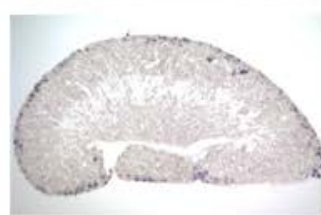

P4

Lhx-1 in-situ hybridization

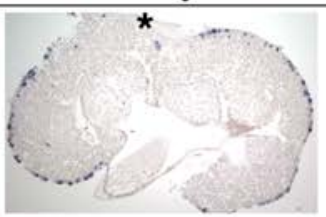

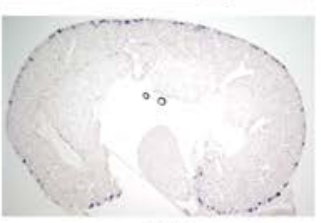

P5

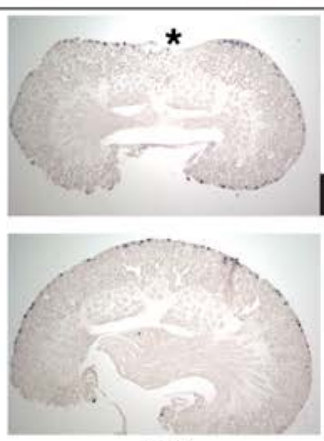

P6
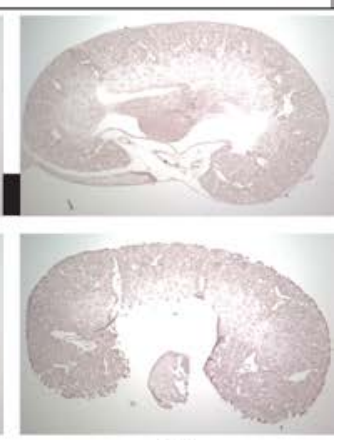

P7

Figure 4. Expression of developmentally regulated genes is unaltered in response to cryoinjury. (A) Immunofluorescence staining for Pax2 and (B) in situ hybridization for Lhx1 were used to determine expression patterns around the injury area compared with contralateral control kidneys. (A) Pax2 expression is not different around injured areas compared with controls (red, Pax2; green, DBA; white, LTL) Original magnification, $\times 20$. (B) Lhx1 is not differentially expressed after injury, as determined by in situ hybridization. Original magnification, $\times 3$. Asterisks indicate site of injury. DBA, Dolichos Biflorus Agglutinin; LTL, Lotus Tetragonolobus Agglutinin.

number associated with low birth weight or nephrons lost to acute or chronic injury is a priority of regenerative medicine in nephrology and would open new avenues for therapy for millions of people.

In contrast to the human, in which de novo nephrogenesis is complete by 36 weeks of gestation, it has been known that there are nephron progenitor cells and immature nephron forms present in the rodent kidney at the normal day of parturition. New nephrons are continuously being generated during kidney development, with the induction of nephron progenitor cells in the cap mesenchyme through interactions with the ureteric bud and the surrounding cortical interstitium. Recent work suggests this niche depends on growth factor signaling pathways (16). This raised the possibility that we might be able to prolong nephrogenesis after parturition by invoking an intrinsic repair response to acute reduction of nephron endowment and learn how we might do so in humans from this response.

The kidney has significant capacity for repair after injury, yet lost nephrons cannot be replaced, leading to decreased function and, together with progressive scarring, end-stage renal disease (35). In rodent models, examples of adaptive regeneration that have been reported to be present exclusively in neonates include replacement of heart tissue (17) and regeneration of fingertips (36) and cochlear cells (37). Importantly, these capabilities are lost with maturation. 


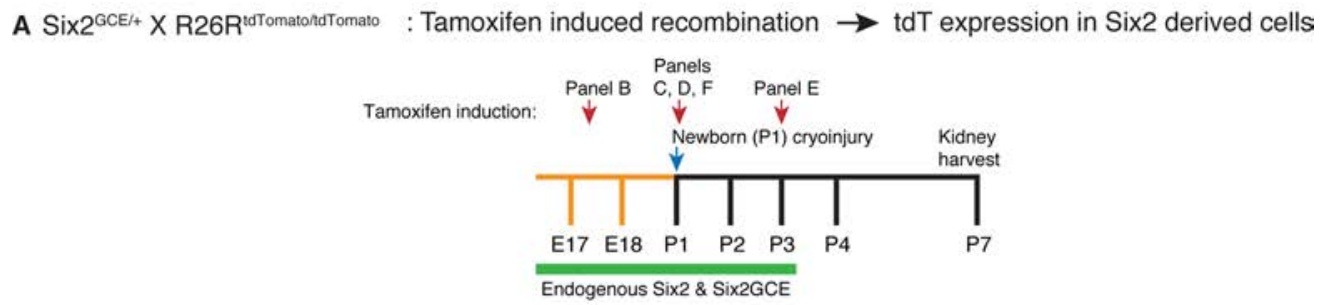

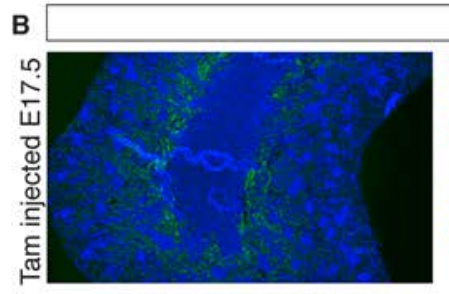

Control - R26R ${ }^{\text {tdomatol/ }}$
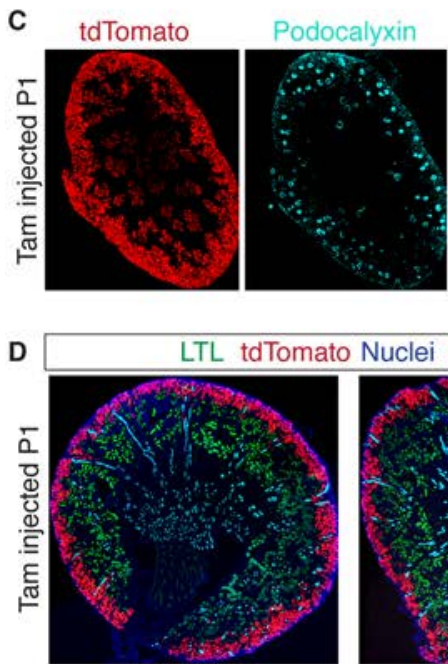

Left injured

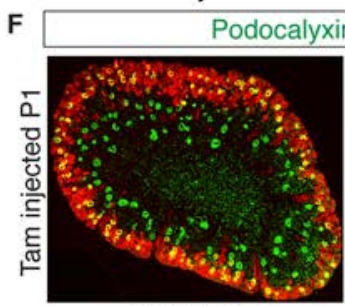

Left injured

\section{LTL, tdTomato, Nuclei}

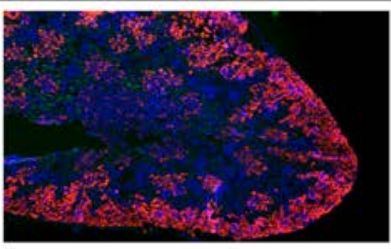

Six $2^{\mathrm{GCE} /+} ; \mathrm{R}^{2} 6 \mathrm{R}^{\text {tdTomato/+ }}$

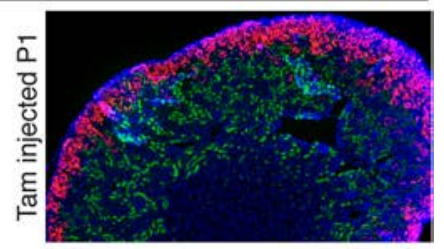

Six $2^{\text {GCE/+; }}$ R26 $\mathrm{R}^{\text {toTomato/t }}$

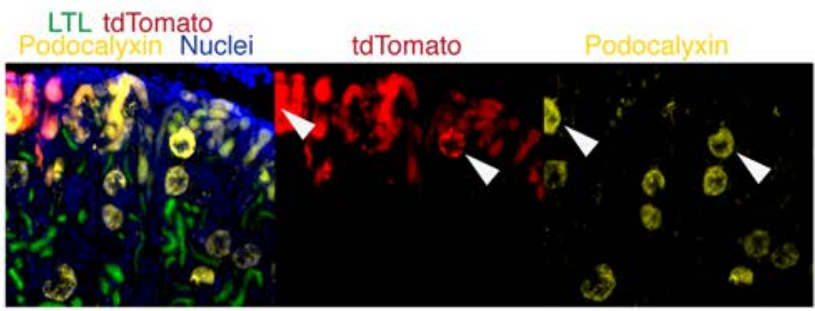

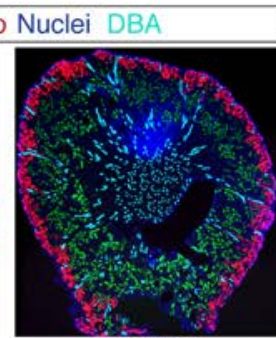

control

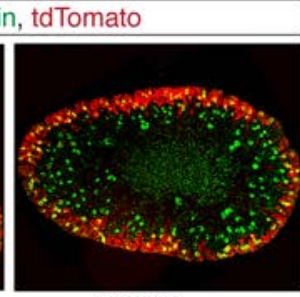

Control
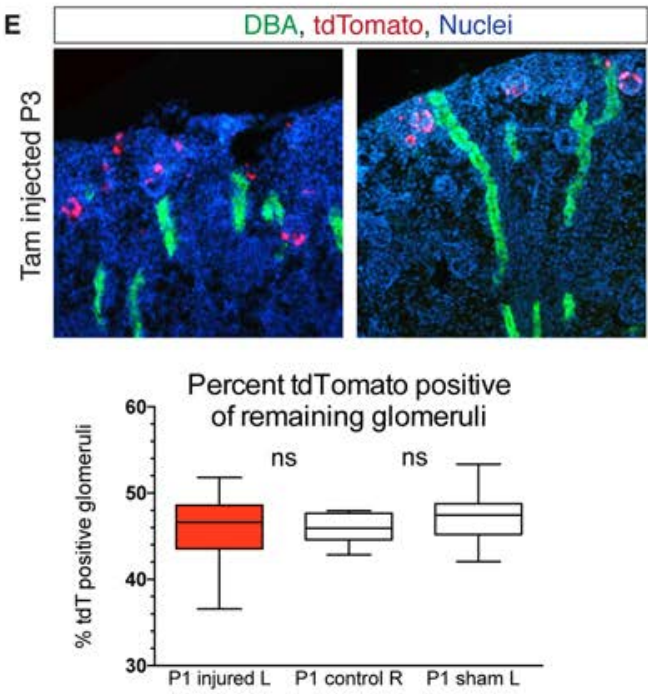

Figure 5. Lineage tracing to determine nephron formation. (A) Breeding strategy of transgenic animals. Six2 heterozygous (Six2 ${ }^{\mathrm{CCE} /+}$ ) mice were $\mathrm{Crossed}$ to tdTomato reporter (R26R ${ }^{\text {tdTomato/tdTomato }}$ ) mice. Injury was induced on the day of birth (P1) followed by tamoxifen injection to induce recombination in Six2 ${ }^{+}$ cells. Kidneys were harvested at P7. tdTomato is expressed permanently in all cells that were Six2 ${ }^{+}$at the time of tamoxifen injection and their daughter cells. (B) Negative controls injected with tamoxifen at E17.5 showed no recombination, as expected (left). Positive controls injected at E17.5 had approximately $50 \%$ of nephrons labeled positive with tdTomato (middle). Animals injected with tamoxifen at P1 had $46 \%$ of glomeruli labeled by tdTomato (right). Original magnification, $\times 4$. (C) Podocalyxin staining in combination with tdTomato fluorescence was used to determine the number of tdTomato-labeled glomeruli as surrogate number for labeled nephrons. An overview of a kidney with the red channel on the left side (tdTomato) and the far-red channel (podocalyxin) on the right side (left). Higher-magnification images showing two dual-labeled (tdTomato and podocalyxin) glomeruli (arrowheads) as well as several non-tdTomato-labeled glomeruli. Original magnification left panel, $\times 4$; right panel, $\times 20$. (D) The percentage of tdTomato-labeled glomeruli was not significantly different in animals subjected to cryoinjury (left) compared with uninjured control animals (right). Original magnification, $\times 4$. (E) When tamoxifen was injected at P3 in animals subjected to cryoinjury, we found only single dispersed tdTomato cells in the outer cortex. Original magnification, $\times 10$. (F) Determination of tdTomato-labeled glomeruli was done using podocalyxin staining (green). Double-labeled glomeruli (podocalyxin and tdTomato) appear yellow (left). The percentage of tdTomato-labeled glomeruli was not significantly different after injury compared with control animals or contralateral control kidneys (right panel). Original magnification, $\times 4 . n=6$ animals per group. In box-and-whisker plots, whiskers depict the minimum and maximum values, boxes depict 25th to 75th percentiles, and lines depict the median. Ordinary 1-way ANOVA, Tukey's multiple comparison test. 
A
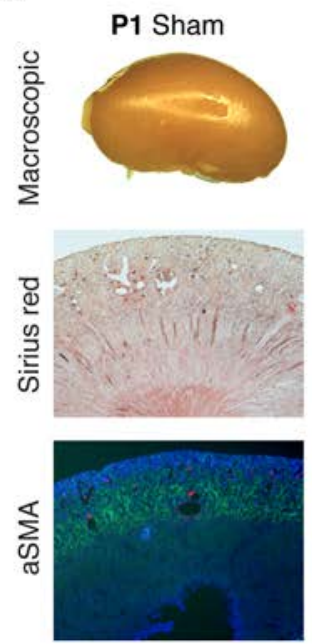

P1 Left cryoinjury
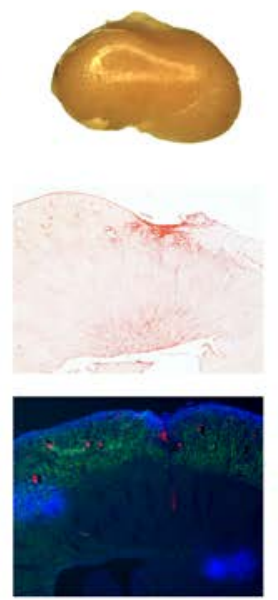

\section{3 weeks}
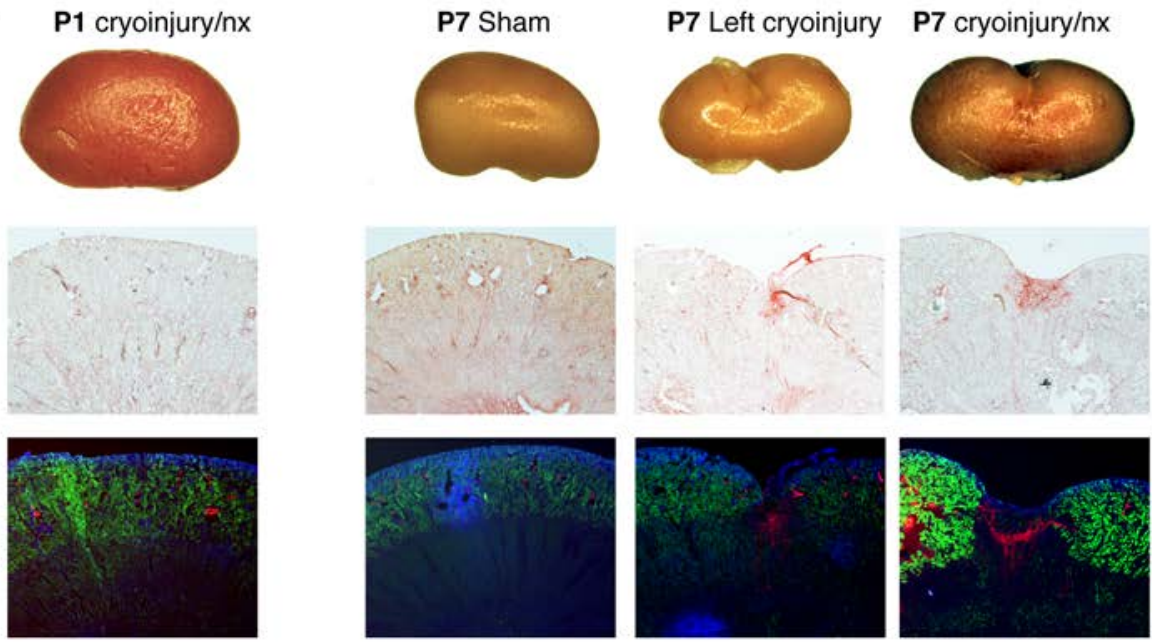

B

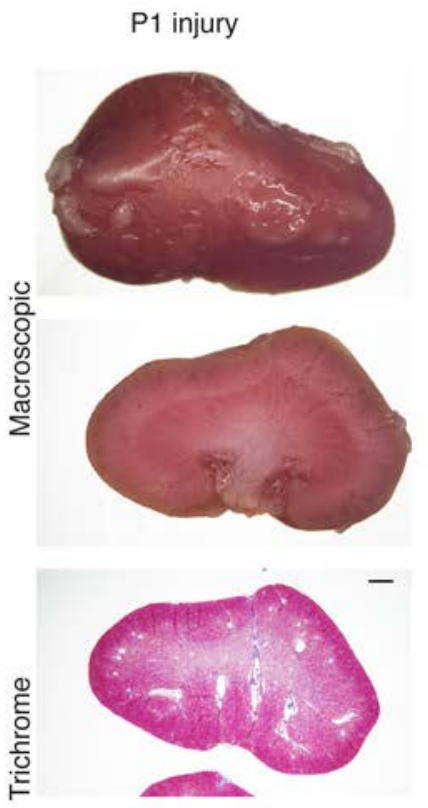

\section{2 weeks}
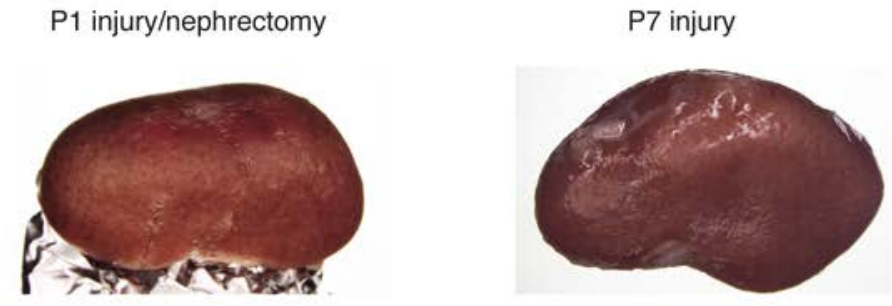

Contralateral control
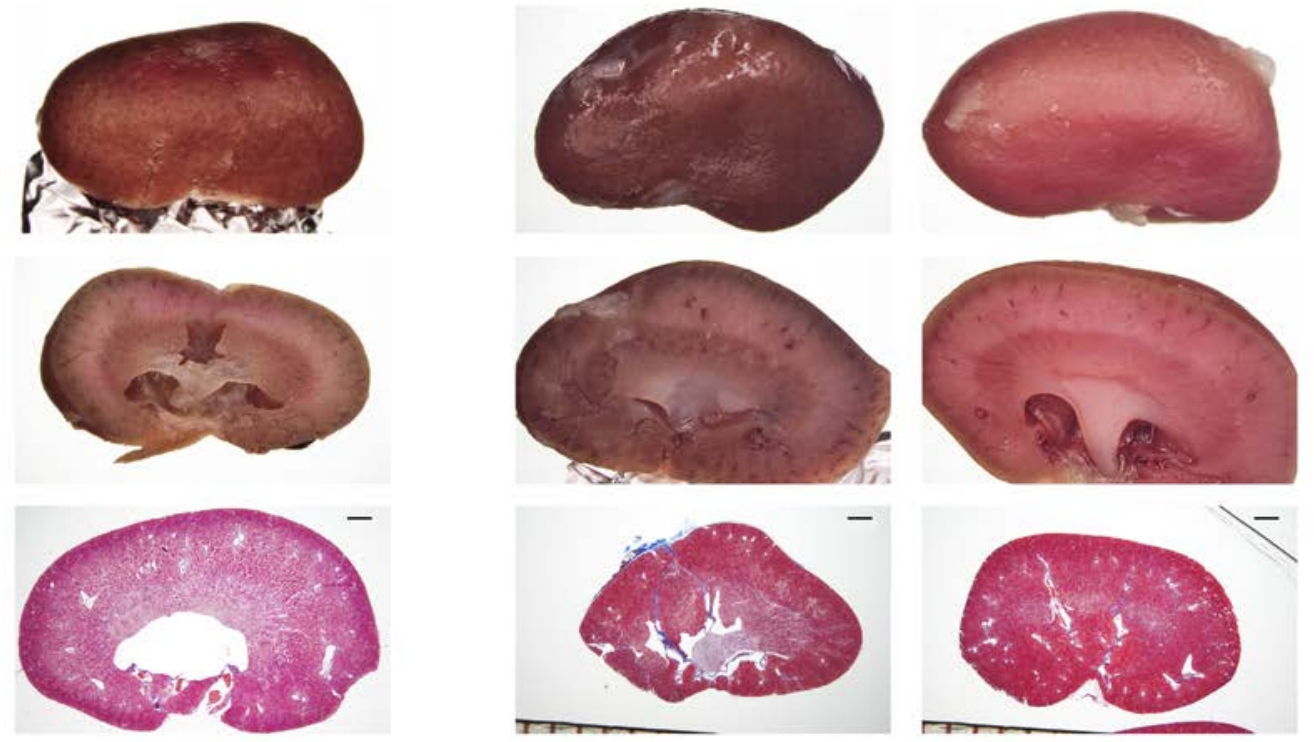

Figure 6. Development of fibrotic lesions after injury. (A) At 3 weeks of age, animals with cryoinjury at P1 showed staining for fibrotic lesions as determined by Sirius red and $\alpha$-SMA, whereas P7 injured animals exhibited more residual indentation and fibrotic lesions at the injury site. Injury with contralateral nephrectomy resulted in healing without fibrosis if injury was carried out on P1 whereas despite nephrectomy fibrosis was present 3 weeks after injury on P7. Original magnification, $\times 3$. (B) Long-term (12 week) follow-up of kidneys subjected to cryoinjury shows indentation and minimal fibrosis at the site of injury in P1-injured animals, with a similar picture in animals with cryoinjury subjected to contralateral nephrectomy. P7-injured animals with cryoinjury had more indentation with fibrotic tissue, as determined by trichrome staining, and this increase was mitigated if there had been contralateral nephrectomy. $n=7$ animals per group. Scale bar: $1.0 \mathrm{~mm}$. SMA, smooth muscle actin.

We devised an injury model to study regeneration in newborn animals using cryoablation to reduce functional kidney tissue and induce reversible injury adjacent to the necrotic cryoinjured area. Newborn mice subjected to cryoinjury recover and heal the injury site, yet we did not find evidence for the generation of new nephrons; rather, we found hypertrophy of existing nephrons to replace lost tissue. While there was some destruction of ureteric bud tips at the injury site, adjacent buds were intact and presumably retained the capacity to induce new nephron formation. We found no difference in proliferative activity adjacent to, or away from, the injury site, and the weight of the injured organ was lower compared with control animals if the contralateral kidney remained in place. When the contralateral kidney was removed at the time of injury, the hypertrophic response of the injured kidney was enhanced such that the weight was greater 
than sham-treated control kidneys at 21 days when normalized by the body weight of the animal. With contralateral nephrectomy, there was no detectable fibrosis 21 days after injury despite the fact that a large part of the kidney had undergone cryoablative necrosis. Though our model results in injury that is localized and creates space for expansion of new nephrons, we did not observe evidence of de novo nephrogenesis.

In approximately one-third of the injured mice we detected the persistence of single and small clusters of Six $2^{+}$cells around the injury area for 2 days longer than in uninjured kidneys. This persistence, however, consisted of single and isolated cells that are unlikely of significance, as they have been separated from an organized niche, and no new nephrons were formed from these dispersed cells. While Six 2 activity is required for maintaining the mesenchymal progenitor population in an undifferentiated state (38), Six2 persistence alone is unlikely to be sufficient for ongoing nephrogenesis. For example, expansion of nephrogenic Six $2^{+}$progenitors by Lin28 overexpression is not sufficient to induce nephrogenesis (39). The lack of formation of new nephrons raises the question of whether the "nephrogenic zone," described to be present at birth in rodents, is capable of creating new nephrons or rather represents a region in which already fate-determined nephrons are maturing. Our data suggest that, in the neonatal mice, Six $2^{+}$cells at birth contribute to approximately $45 \%$ of glomeruli, but the ability to expand this population and produce additional glomeruli is lost and is not enhanced after injury.

Given the long-term adverse outcomes associated with low nephron number at birth, with increased incidence of hypertension and chronic kidney disease (30), it is important to understand the determinants of nephrogenesis with an eye to developing interventions that can enhance nephron number. New nephrons can be generated in response to partial nephrectomy in adult fish (40) but, to date, attempts to do so in adult mammals have been unsuccessful. Importantly, one study using in utero partial renal ablation in an ovine model showed increased nephron numbers in the contralateral kidney after the removal of one kidney (41). The stage of nephrectomy targeted in this study was well before the completion of kidney formation and thus underlines the importance of timing in inducing additional nephron formation. Our work targeted late-stage nephrogenesis in mice. It is not clear why new nephron formation does not continue after P3 or cannot be induced at P1-P3 in mice with an intact "nephrogenic zone." Gene expression patterns regulating interactions between early nephrons and the ureteric epithelium are altered by P2 $(12,13)$, and there is strong expression of TMEM100, a proximal tubule marker, in the cap mesenchyme by P1 (42). Rumballe et al. (13) have proposed an accelerated differentiation at P1. These observations, together with our own, support a model in which the cap mesenchyme has already committed to nephron formation by P1.

In conclusion, our data demonstrate that, despite a persistence of a "nephrogenic zone" at birth, newborn mouse kidneys are unable to generate compensatory new nephrons in response to acute kidney mass reduction. In the rodent, the capability for nephrogenesis postnatally in response to nephron reduction appears to be limited to the nephrons that are already at a committed stage of development by the time of birth.

\section{Methods}

Cryoablation surgery. Outbred CD1 wild-type mice were obtained pregnant from the vendor (Charles River), and mice were left to litter naturally. The morning a new litter was observed was considered the first postnatal day and was labeled "P1," with subsequent days counted as P2 and up. Neonatal pups within 24 hours of birth (P1), a time at which the nephrogenic zone and Six $2^{+}$progenitors are present in the renal cortex, were subjected to cryoinjury, as described as follows. Mice were placed inside a latex glove to keep dry and anesthetized by immersion into ice water for 5 minutes, which causes reversible apnea and asystole, thereby preventing excessive blood loss during surgery. The complete procedure was performed on an ice block to prevent rewarming during surgery. Lateral flank incisions were performed to expose the kidneys.

For cryoinjury, the kidney was gently pulled out of the retroperitoneal space with a cotton swap. A cooling probe, consisting of the tip of a blunt steel framing nail placed into a syringe outlet, cooled by liquid nitrogen placed inside the syringe, was placed for 3 seconds on the kidney surface at the middle of the kidney curvature. The cooling probe produced a consistent and reproducible area of injury that was initially white in appearance while cold but quickly returned to the color of the remaining kidney.

For nephrectomy, the kidney was exposed after a flank incision and gently pulled out, and 6.0 silk suture was placed around the kidney. After closure of the suture around the kidney, the parenchyma was cut with scissors to remove the whole kidney parenchyma, leaving the closed suture in place to prevent bleeding.

Following cryoinjury and/or nephrectomy, the flank incisions were sutured with two 6.0 silk closure 
sutures in one layer, and the mice were warmed slowly under a warming lamp. The return of blood circulation was visible by a change in skin color to pink and movement of the animals. Sham operated mice underwent the same procedure, except the kidneys were exposed without cryoinjury. The entire procedure lasted 10-15 minutes depending on the extend of the procedure (sham versus cryoinjury versus cryoinjury plus nephrectomy). After refinement of the technique and minimization of bleeding, more than $90 \%$ of animals survived the surgical procedure immediately after anesthesia. Overall survival was lower because of maternal cannibalization of about $10 \%$ of pups after placing pups back with the mother. Surgical mortality was similar when carried out in neonates or P7 animals.

The same anesthesia protocol was employed for kidney pole resection. After placement of a suture around the lower pole, microsurgery scissors were applied to the pole of the kidney to remove $10 \%-15 \%$ of the kidney. Kidneys were harvested 5 weeks after resection. The data presented in Figure $2 \mathrm{G}$ are from 10 animals pooled from 3 separate experimental dates.

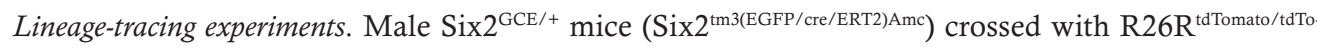

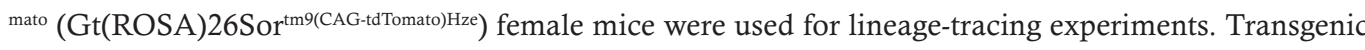
mice were obtained from Jackson laboratory and bred and mated in the local animal facility. Induction of Cre recombinase activity and reporter gene expression was achieved by administering a single dose of $0.2 \mathrm{mg}(\sim 0.05 \mathrm{mg} / \mathrm{g} \mathrm{BW})$ estrogen analog tamoxifen (Sigma-Aldrich) dissolved in corn oil subcutaneously to neonatal mice either immediately after surgery on P1 or, in a subset of animals, at P4 to lineage-trace Six $2^{+}$cells. Mice with the Six ${ }^{\mathrm{GCE}}$ allele express a GFP::CreERt2 fusion protein in the Six2 expression domain (27). This protein remains sequestered outside the nucleus until tamoxifen is administered. Upon tamoxifen treatment, the GFP::CreERt2 protein translocates to the nucleus, acting on the loxP sites flanking a STOP sequence upstream of the tdTomato gene insert, resulting in permanent red (tdTomato RFP) labeling of all cells that express Six 2 at the time of tamoxifen administration.

For frozen sections, tissues were fixed in $4 \%$ paraformaldehyde in PBS for 1 hour at $4^{\circ} \mathrm{C}$, dehydrated using 30\% sucrose overnight and snap-frozen in OCT. $20-\mu \mathrm{m}$-thick sections were prepared using a cryotome for immunofluorescence and in situ hybridizations.

Tissue preparation. For H\&E and trichrome staining, paraffin embedding and sectioning $(5 \mu \mathrm{m})$ was performed as follows. Kidneys were fixed overnight in $10 \%$ formalin at room temperature, moved to $70 \%$ ethanol, and stored up to 1 week before paraffin embedding. H\&E and Masson's trichrome staining were performed according to standard procedures.

Immunofluorescence. Frozen sections (20 $\mu \mathrm{m}$ thickness) were warmed, dried, and washed in PBS/0.1\%Triton (PBT) and blocked for 1 hour with 5\% donkey serum. The primary antibody was applied at $4{ }^{\circ} \mathrm{C}$ overnight at the tested dilution (in PBT and 1\% donkey serum). Following 3 washes with PBT, the secondary antibody was applied together, at times, with either Lotus Tetragonolobus Agglutinin (LTL) (Vector labs, B-1325) or Dolichos Biflorus Agglutinin (DBA) (Vector labs, FL-1031) for 2 hours, followed by a brief rinse with PBT and nuclear stain with Hoechst or DAPI for 10 minutes. Mounting was done with Vectashield (Vector labs). Primary antibodies used were as follows: rabbit polyclonal anti-Six2 (Proteintech, 11562-1-AP) and Pax2 (Abcam, ab37129).

In situ hybridization. Frozen sections (20 $\mu \mathrm{m}$ thickness) of mouse kidneys were hybridized with digoxigenin-labeled antisense riboprobes generated using gene-specific PCR. The complete protocol for riboprobe generation and section in situ hybridization is available on the GUDMAP web site (http:// gudmap.org/) listed under "Protocols" $(43,44)$. In situ hybridization was performed after thawing and drying sections stored at $-80^{\circ} \mathrm{C}$. Sections were fixed in $4 \%$ PFA for 10 minutes and washed in PBS 3 times. Incubation with $10 \mu \mathrm{g} / \mathrm{ml}$ of proteinase- $\mathrm{K}$ was followed by 3 washes in PBS. After proteinase $\mathrm{K}$ treatment, samples were fixed again and prehybridized at $70^{\circ} \mathrm{C}$ for 1 hour before being hybridized with $500 \mathrm{ng} / \mathrm{ml}$ digoxigenin-labeled riboprobes at $70^{\circ} \mathrm{C}$ overnight. Samples were washed with hot solutions at $65^{\circ} \mathrm{C}$ and treated with $2 \mu \mathrm{g} / \mathrm{ml}$ RNase A for 15 minutes. After additional stringent hot washes, samples were washed with $1 \times$ MBST $(0.1 \mathrm{M}$ maleic acid, $0.15 \mathrm{M} \mathrm{NaCl}, 0.1 \%$ Tween-20 [pH 7.5]), blocked in blocking solution (20\% heat-inactivated sheep serum in MBST plus 2\% Boehringer Mannheim Blocking Reagent), and incubated subsequently with anti-digoxigenin antibody conjugated with alkaline phosphatase (Roche, 1:4,000) overnight at $4^{\circ} \mathrm{C}$. Extensive washing in $1 \times \mathrm{MBST}$ was followed by incubation in NTMT (100 mM NaCl, 100 mM Tris- $\mathrm{HCl}$ [pH 9.5], $50 \mathrm{mM} \mathrm{MgCl}$, 0.1\% Tween-20, 2 mM levamisole). Hybridization signal was visualized by adding BM purple (Roche). Color reactions were observed at different time points and terminated when signals were strong or a background developed. 
After development of the colorimetric assay, samples were post-fixed and stored in $80 \%$ glycerol/PBS. Images were then captured with a Nikon digital camera (Nikon) attached to a Nikon SMZ1500 stereoscope (Nikon).

Quantitative analysis of proliferation. Frozen sections were stained with anti-Ki67 (Abcam) and LTL. $\mathrm{Ki} 67^{+}$cells were counted in $\times 40$ sections taken from 3 different randomly chosen areas in the mid-cortex, both close to and remote from injury site.

Glomerular counting. The acid maceration method was used to determine glomerular number. Acid maceration to quantitate glomerular number is a commonly used and readily accessible method for estimating glomerular number, and the advantages are the ease of performance, low cost, and rapidity. Sources of error are potential counting errors and low precision associated with hemocytometer use or nonuniform suspension. More precise methods, such as the common gold standard, the unbiased physical disector/fractionator method, are extremely labor and cost intensive, because a whole kidney is sectioned exhaustively. We believe that acid maceration is reasonably accurate in estimating the glomerular number in a whole kidney, and it is commonly used in the literature as a standard method to estimate nephron number (23). Briefly, harvested kidneys were decapsulated and chopped into small pieces with a razor blade and then suspended in $1 \mathrm{ml}$ of $6 \mathrm{~N} \mathrm{HCl}$ at $37^{\circ} \mathrm{C}$ for 1 hour, followed by trituration by micropipette. Five milliliters of distilled water were added to a total volume of $6 \mathrm{ml}$, which was incubated overnight at $4^{\circ} \mathrm{C}$. Pictures of $10-\mu 1$ sample volumes on a Neubauer chamber with grid (without using a coverslip) were taken on a Nikon SMZ1500 stereoscope. For each sample, a total of 10 pictures were taken for counting, so that a volume of $100 \mu 1$ was counted. Manual counting was done blinded to the sample using ImageJ (NIH) (45).

Statistics. Statistical analyses were performed using GraphPad Prism 5 software. Significance was tested using the 2-tailed unpaired Student's $t$ test or 1-way ANOVA with Dunnett or Tukey analysis for multiple comparisons when appropriate. A $P$ value of less than 0.05 was considered statistically significant. All values are expressed as mean $\pm \mathrm{SD}$.

Study approval. All protocols were approved by the Institutional Animal Care and Use Committee of the Harvard Medical Area Standing Committee on Animals, Harvard Medical School.

\section{Author contributions}

JVB, FT, BSF, and RI conceived the study. JVB, FT, RI, BSF, MTV, and MG provided study methodology. FT, RI, BSF, and MTV performed experiments. JVB, MTV, and MG provided resources. JVB acquired study funding. FT and MTV wrote the original draft of the manuscript. FT, BSF, MTV, and JVB reviewed and edited the manuscript.

\section{Acknowledgments}

This work was supported by NIH grants R01DK072381 and R37DK039773 (to JVB), T32DK007527 (to FT), and K01DK102826 and F32DK092036 (to BSF) and the Harvard Stem Cell Institute and U01DK107350 (NIH sub award) (to MTV). The content is solely the responsibility of the authors and does not necessarily represent the official views of the NIH.

Address correspondence to: Joseph V. Bonventre or M. Todd Valerius, 4 Blackfan Circle, Harvard Institute of Medicine, Boston, Massachusetts 02115, USA. Phone: 617.525.5960; E-mail: joseph_bonventre@hms.harvard. edu (J.V. Bonventre). Phone: 617.525.4030; E-mail: mtvalerius@research.bwh.harvard.edu (M.T. Valerius).

BSF's present address is: Division of Nephrology, Kidney Research Institute, and Institute for Stem Cell and Regenerative Medicine, Department of Medicine, University of Washington School of Medicine, Seattle, Washington, USA.

RI's present address is: Vita-Salute San Raffaele University, IRCCS San Raffaele Scientific Institute, Milan, Italy.

\footnotetext{
1. Hinchliffe SA, Sargent PH, Howard CV, Chan YF, van Velzen D. Human intrauterine renal growth expressed in absolute number of glomeruli assessed by the disector method and Cavalieri principle. Lab Invest. 1991;64(6):777-784.

2. Faa G, et al. Marked interindividual variability in renal maturation of preterm infants: lessons from autopsy. J Matern Fetal Neonatal Med. 2010;23 Suppl 3:129-133.

3. Hayman JM. Renal function and the number of glomeruli in the human kidney. Arch Intern Med. 1939;64(1):69-83.

4. Hughson M, Farris AB, Douglas-Denton R, Hoy WE, Bertram JF. Glomerular number and size in autopsy kidneys: the rela-
} 
tionship to birth weight. Kidney Int. 2003;63(6):2113-2122.

5. Kandasamy Y, Smith R, Wright IM, Lumbers ER. Relationships between glomerular filtration rate and kidney volume in lowbirth-weight neonates. J Nephrol. 2013;26(5):894-898.

6. Barker DJ, Bull AR, Osmond C, Simmonds SJ. Fetal and placental size and risk of hypertension in adult life. BMJ. 1990;301(6746):259-262.

7. Keller G, Zimmer G, Mall G, Ritz E, Amann K. Nephron number in patients with primary hypertension. $N$ Engl J Med. 2003;348(2):101-108.

8. Hughson MD, Douglas-Denton R, Bertram JF, Hoy WE. Hypertension, glomerular number, and birth weight in African Americans and white subjects in the southeastern United States. Kidney Int. 2006;69(4):671-678.

9. Schreuder MF, Langemeijer ME, Bökenkamp A, Delemarre-Van de Waal HA, Van Wijk JA. Hypertension and microalbuminuria in children with congenital solitary kidneys. J Paediatr Child Health. 2008;44(6):363-368.

10. Luyckx VA, Brenner BM. The clinical importance of nephron mass. J Am Soc Nephrol. 2010;21(6):898-910.

11. White SL, et al. Is low birth weight an antecedent of CKD in later life? A systematic review of observational studies. Am J Kidney Dis. 2009;54(2):248-261

12. Hartman HA, Lai HL, Patterson LT. Cessation of renal morphogenesis in mice. Dev Biol. 2007;310(2):379-387.

13. Rumballe BA, Georgas KM, Combes AN, Ju AL, Gilbert T, Little MH. Nephron formation adopts a novel spatial topology at cessation of nephrogenesis. Dev Biol. 2011;360(1):110-122.

14. Charlton JR, Springsteen CH, Carmody JB. Nephron number and its determinants in early life: a primer. Pediatr Nephrol. 2014;29(12):2299-2308

15. Hayslett JP. Effect of age on compensatory renal growth. Kidney Int. 1983;23(4):599-602.

16. Brown AC, Muthukrishnan SD, Oxburgh L. A synthetic niche for nephron progenitor cells. Dev Cell. 2015;34(2):229-241.

17. Porrello ER, et al. Transient regenerative potential of the neonatal mouse heart. Science. 2011;331(6020):1078-1080

18. Andersen DC, Ganesalingam S, Jensen CH, Sheikh SP. Do neonatal mouse hearts regenerate following heart apex resection? Stem Cell Reports. 2014;2(4):406-413.

19. Goss RJ. Similarities and differences between mechanisms of organ and tissue growth regulation. Proc Nutr Soc. 1990;49(3):437-442

20. Han WK, Bailly V, Abichandani R, Thadhani R, Bonventre JV. Kidney Injury Molecule-1 (KIM-1): a novel biomarker for human renal proximal tubule injury. Kidney Int. 2002;62(1):237-244.

21. Roth J. Application of lectin--gold complexes for electron microscopic localization of glycoconjugates on thin sections. $J$ Histochem Cytochem. 1983;31(8):987-999.

22. Brunskill EW, Lai HL, Jamison DC, Potter SS, Patterson LT. Microarrays and RNA-Seq identify molecular mechanisms driving the end of nephron production. BMC Dev Biol. 2011;11:15.

23. Cebrian C, Asai N, D'Agati V, Costantini F. The number of fetal nephron progenitor cells limits ureteric branching and adult nephron endowment. Cell Rep. 2014;7(1):127-137.

24. Bertram JF. Estimating glomerular number: why we do it and how. Clin Exp Pharmacol Physiol. 2013;40(11):785-788

25. Harshman LA, Brophy PD. PAX2 in human kidney malformations and disease. Pediatr Nephrol. 2012;27(8):1265-1275.

26. Cirio MC, et al. Lhx1 is required for specification of the renal progenitor cell field. PLoS One. 2011;6(4):e18858.

27. Kobayashi A, et al. Six 2 defines and regulates a multipotent self-renewing nephron progenitor population throughout mammalian kidney development. Cell Stem Cell. 2008;3(2):169-181.

28. Short KM, et al. Global quantification of tissue dynamics in the developing mouse kidney. Dev Cell. 2014;29(2):188-202.

29. Al-Awqati Q, Oliver JA. Stem cells in the kidney. Kidney Int. 2002;61(2):387-395.

30. Luyckx VA, et al. Effect of fetal and child health on kidney development and long-term risk of hypertension and kidney disease. Lancet. 2013;382(9888):273-283.

31. Morizane R, Lam AQ, Freedman BS, Kishi S, Valerius MT, Bonventre JV. Nephron organoids derived from human pluripotent stem cells model kidney development and injury. Nat Biotechnol. 2015;33(11):1193-1200.

32. Freedman BS, et al. Modelling kidney disease with CRISPR-mutant kidney organoids derived from human pluripotent epiblast spheroids. Nat Commun. 2015;6:8715.

33. Takasato M, et al. Kidney organoids from human iPS cells contain multiple lineages and model human nephrogenesis. Nature. 2015;526(7574):564-568.

34. Morizane R, Bonventre JV. Generation of nephron progenitor cells and kidney organoids from human pluripotent stem cells. Nat Protoc. 2017;12(1):195-207.

35. Humphreys BD. Kidney injury, stem cells and regeneration. Curr Opin Nephrol Hypertens. 2014;23(1):25-31.

36. Han M, Yang X, Lee J, Allan CH, Muneoka K. Development and regeneration of the neonatal digit tip in mice. Dev Biol. 2008;315(1):125-135.

37. Cox BC, et al. Spontaneous hair cell regeneration in the neonatal mouse cochlea in vivo. Development. 2014;141(4):816-829.

38. Self M, et al. Six2 is required for suppression of nephrogenesis and progenitor renewal in the developing kidney. $E M B O J$. 2006;25(21):5214-5228.

39. Urbach A et al. Lin28 sustains early renal progenitors and induces Wilms tumor. Genes Dev. 2014;28(9):971-982.

40. Elger M, et al. Nephrogenesis is induced by partial nephrectomy in the elasmobranch Leucoraja erinacea. J Am Soc Nephrol. 2003;14(6):1506-1518.

41. Douglas-Denton R, Moritz KM, Bertram JF, Wintour EM. Compensatory renal growth after unilateral nephrectomy in the ovine fetus. J Am Soc Nephrol. 2002;13(2):406-410.

42. Georgas K, et al. Analysis of early nephron patterning reveals a role for distal RV proliferation in fusion to the ureteric tip via a cap mesenchyme-derived connecting segment. Dev Biol. 2009;332(2):273-286.

43. Yu J, et al. Identification of molecular compartments and genetic circuitry in the developing mammalian kidney. Development. 2012;139(10):1863-1873.

44. McMahon Group Protocols. GUDMAP Genitourinary Development Molecular Anatomy Project. http://www.gudmap.org/ Research/Protocols/McMahon.html. Accessed January 17, 2017.

45. Schneider CA, Rasband WS, Eliceiri KW. NIH Image to ImageJ: 25 years of image analysis. Nat Methods. 2012;9(7):671-675. 